\title{
Fusion patterns of major calvarial sutures on volume-rendered CT reconstructions
}

\author{
C. Corbett Wilkinson, MD, ${ }^{1}$ Nicholas V. Stence, MD, ${ }^{2}$ Cesar A. Serrano, MD, ${ }^{3}$ Sarah J. Graber, BA, ${ }^{1}$ \\ Lígia Batista-Silverman, MA, ${ }^{1}$ Emily Schmidt-Beuchat, MD, ${ }^{4}$ and Brooke M. French, MD $^{5}$
}

\begin{abstract}
Departments of ${ }^{1}$ Neurosurgery, ${ }^{2}$ Radiology, and ${ }^{5}$ Plastic Surgery, Children's Hospital Colorado, University of Colorado Denver, Anschutz Medical Campus, Aurora, Colorado; ${ }^{3}$ Department of Neurosurgery, West Virginia University, Morgantown, West Virginia; and ${ }^{4}$ Department of Obstetrics, Gynecology, and Reproductive Science, Icahn School of Medicine at Mount Sinai, New York, New York
\end{abstract}

\begin{abstract}
OBJECTIVE Recently, the authors investigated the normal course of fusion of minor lateral calvarial sutures on "3D" volume-rendered head CT reconstructions in pediatric trauma patients. While evaluating these reconstructions, they found many more fused sagittal sutures than expected given the currently accepted prevalence of sagittal craniosynostosis. In the present study, using the same set of head CT reconstructions, they investigated the course of fusion of the sagittal as well as the lambdoid, coronal, and metopic sutures.
\end{abstract}

METHODS They reviewed all volume-rendered head CT reconstructions performed in the period from 2010 through mid-2012 at Children's Hospital Colorado for trauma patients aged 0-21 years. Each sagittal, lambdoid, coronal, or metopic suture was graded as open, partially fused, or fused. The cephalic index $(\mathrm{Cl})$ was calculated for subjects with fused and partially fused sagittal sutures.

RESULTS After exclusions, 331 scans were reviewed. Twenty-one subjects (6\%) had fusion or partial fusion of the sagittal suture. Four of the 21 also had fusion of the medial lambdoid and/or coronal sutures. In the 17 subjects (5\%) with sagittal suture fusion and no medial fusion of adjacent sutures, the mean $\mathrm{Cl}$ was 77.6. None of the 21 subjects had been previously diagnosed with craniosynostosis. Other than in the 21 subjects already mentioned, no other sagittal or lambdoid sutures were fused at all. Nor were other coronal sutures fused medially. Coronal sutures were commonly fused inferiorly early during the 2 nd decade of life, and fusion progressed superiorly and medially as subjects became older; none were completely fused by 18 years of age. Fusion of the metopic suture was first seen at 3 months of life; fusion was often not complete until after 2 years.

CONCLUSIONS The sagittal and lambdoid sutures do not usually begin to fuse before 18 years of age. However, more sagittal sutures are fused before age 18 than expected given the currently accepted prevalence of craniosynostosis. This finding is of unknown significance, but likely many of them do not need surgery. The coronal suture often begins to fuse inferiorly early in the 2 nd decade of life but does not usually complete fusion before 18 years of age. The metopic suture often starts to fuse by 3 months of age, but it may not completely fuse until after 2 years of age.

https://thejns.org/doi/abs/10.3171/2019.11.PEDS1953

KEYWORDS craniosynostosis; metopic suture; coronal suture; sagittal suture; lambdoid suture; craniofacial; congenital

$\mathrm{C}$ RANIOSYNOSTOSIS is estimated to occur in about 1 per 2100-2325 live births, ${ }^{3,9}$ with sagittal synostosis being the most common type at 1 in 5250-6667 live births. ${ }^{3,10}$ Recently, we investigated the fusion of minor lateral calvarial sutures (sphenoparietal, squamosal, parietomastoid) on the volume-rendered head CT reconstructions in 337 pediatric trauma patients. While reviewing these reconstructions, we discovered many more fused sagittal sutures than the expected 1 in 5000-6000 sub- jects. In the present study, using the same head CT reconstructions, we investigated abnormal fusion of the sagittal as well as the lambdoid, coronal, and metopic sutures.

Several publications have reviewed normal fusion of the metopic suture on CT.,12,15,22,23 However, there is less information on normal fusion of other major sutures. Most existing information derives from studies of adult cadaveric skulls, ${ }^{2,5,14,16-21}$ although there is at least one recent CT study in the forensic literature. ${ }^{6}$ With the present publica- 
tion, we hope to increase the information available about the normal fusion of major calvarial sutures.

\section{Methods}

In 2010, Children's Hospital Colorado began performing most head CTs as spiral scans, allowing high-resolution, "3D" volume-rendered reconstructions to be made of the skull. That same year, Children's Colorado began performing the initial head CT for all trauma patients using a new protocol, "CT brain without contrast for trauma," which includes volume-rendered reconstructions. All scans in this study were obtained using this protocol on a Siemens SOMATOM Definition Flash CT scanner. Scans were acquired helically with a slice thickness of $0.5 \mathrm{~mm}$. Volume-rendered reconstructions of the skull were created by the CT technologist for each case. Under a Colorado Multiple Institutional Review Board protocol, we reviewed all initial volume-rendered head CT reconstructions performed at Children's Colorado in the period from January 2010 through mid-2012 for trauma patients aged $0-21$ years.

Each sagittal, lambdoid, coronal, and metopic suture in every reconstruction was evaluated independently by a board-certified pediatric neuroradiologist (N.V.S.) and pediatric neurosurgeon (C.C.W.). Each suture was graded as open, partially fused, or fused. A length of suture was considered fused when no discernible suture was seen over that length on a volume-rendered reconstruction of the outer skull surface. A suture length was considered open when it did contain clearly visible suture. A suture was considered partially fused when any length except its entire length was fused. When no length of the suture was fused, the suture was called "open"; when the entire length was fused, the suture was called "fused." When there was a discrepancy between the grades assigned to a particular suture by the two reviewers, the reviewers reevaluated the suture jointly and assigned a consensus grade. When it was difficult to determine the extent of fusion of certain sutures and when it was suspected that sutures were fused early and/or atypically, we also evaluated the source images. Although we consider each normal cranium to have two coronal and two lambdoid sutures, left and right for each, for this study, we considered each cranium to have a single bilateral coronal and a single bilateral lambdoid suture.

Individual sutures were excluded if they were obscured by artifact, incompletely imaged, imaged with insufficient quality to evaluate their extent of fusion, or directly involved by fracture. If there was a fracture elsewhere in the calvaria that did not directly involve a particular suture, that suture was still included. All sutures were excluded when no volume-rendered reconstructions were available or when the available reconstructions were of insufficient quality, when the subject had a cerebrospinal fluid shunt, or when the subject had radiological evidence of previous cranial or intracranial surgery or significant abnormalities of the brain.

The only records reviewed for most subjects were their head CT images. However, for subjects with fused or partially fused sagittal sutures, we also searched lists of exist- ing diagnoses for craniosynostosis. Only one CT scan was reviewed per subject.

For subjects with fused or partially fused sagittal sutures, the cephalic index (CI) was calculated from their CT scans. To calculate the CI, the distance between the left and right euryon was divided by the distance between the glabella and opisthocranion and multiplied by 100 . The percentages of sutures that were open, partially fused, or fused were then tabulated and graphed by month and year of age. The degree of suture fusion was tabulated by month from 1 month through 36 months of age, rounding up or down to the nearest whole month. After the age of 36 months, fusion was tabulated only by year. For ages 1 and 2 years, we evaluated the scans of all subjects aged 9 through 15 months and 21 through 27 months, respectively. For the age of 3 years, we evaluated the scans of all subjects aged 33 through 42 months. For the age of 4 years and older, all subjects were included and their ages were rounded up or down to the nearest whole year.

For statistical analysis, the data were organized and analyzed using JMP (JMP Pro, version 12, SAS Institute Inc.). Comparisons were made using Wilcoxon's rank-sum test (continuous variables) and Fisher's exact test (categorical variables). Significance was set at $\mathrm{p}<0.05$.

\section{Results}

We initially reviewed 337 volume-rendered head CT reconstructions. Two subjects were excluded because of preexisting ventriculoperitoneal shunts. Two were excluded because of an age $>21$ years ( 30 and 31 years). As there were only two 19-year-olds (and no 20- or 21-year-olds), we excluded them too. One coronal suture was excluded because of involvement by fracture. Thus, 331 reconstructions and 1323 sutures were ultimately included (Supplemental Table 1). The subject ages ranged from 11 days to 18 years. There were 215 males (65\%) and 116 females (35\%).

The sagittal suture was fused or partially fused in 21 subjects (6\%; Table 1). In 4 subjects, the medial sections of adjacent sutures were also fused (Fig. 1D-G): the lambdoid in 2 , the coronal in 1 , and the lambdoid and coronal in 1 . None of these 21 subjects were found to have preexisting diagnoses of craniosynostosis.

Other than in the aforementioned 21 subjects, no sagittal or lambdoid sutures were even minimally fused and no coronal sutures were fused medially. Medial lambdoid fusion occurred in $0.9 \%$ of subjects and medial coronal fusion in $0.6 \%$ (Table 1). Several older subjects had narrow sutures with substantial ridging that were still open throughout their lengths (Fig. 2A). This pattern was called "lapsed suture union" by Todd and Lyon. ${ }^{20}$

Of all subjects with sagittal suture fusion, whether complete or partial, $17(81 \%)$ were male and $4(19 \%)$ were female (Table 2). After adjustment for the proportion of males $(65 \%)$ versus females $(35 \%)$ in the entire series, there were $70 \%$ males and $30 \%$ females. Of the subjects with isolated sagittal suture fusion (without medial fusion of adjacent sutures), 14 (82\%) were male and $3(18 \%)$ were female. After adjustment for the proportion of males versus females in the entire series, there were $72 \%$ males and $28 \%$ females. Of the subjects with medial fusion of 
TABLE 1. Clinical data of subjects with fusion or partial fusion of sagittal suture, with or without medial fusion of adjacent sutures

\begin{tabular}{|c|c|c|c|c|c|c|}
\hline Case No. & Age (yrs) & Sex & Sutures Fused & $\begin{array}{c}\text { Complete vs Partial Fusion of } \\
\text { Sagittal Suture }\end{array}$ & $\begin{array}{l}\text { Location of Open } \\
\text { Suture Section* }\end{array}$ & Cephalic Index \\
\hline 1 & 2 & M & Sagittal & Partial & Ant & 88 \\
\hline 2 & 2 & M & Sagittal & Complete & & 71 \\
\hline 3 & 3 & M & Sagittal & Partial & Ant & 83 \\
\hline 4 & 3 & M & Sagittal & Partial & Ant/pst & 75 \\
\hline 5 & 4 & M & Sagittal & Partial & Ant/pst & 72 \\
\hline 6 & 5 & M & Sagittal, coronal & Complete & & 96 \\
\hline 7 & 5 & M & Sagittal & Partial & Ant & 76 \\
\hline 8 & 5 & M & Sagittal, coronal, lambdoid & Complete & & 74 \\
\hline 9 & 8 & M & Sagittal & Complete & & 76 \\
\hline 10 & 9 & $\mathrm{~F}$ & Sagittal, lambdoid & Complete & & 79 \\
\hline 11 & 9 & M & Sagittal & Complete & & 75 \\
\hline 12 & 10 & M & Sagittal & Complete & & 87 \\
\hline 13 & 11 & M & Sagittal & Partial & Ant/pst & 84 \\
\hline 14 & 11 & M & Sagittal & Complete & & 76 \\
\hline 15 & 12 & $\mathrm{~F}$ & Sagittal & Partial & Ant/pst & 84 \\
\hline 16 & 12 & $\mathrm{~F}$ & Sagittal & Complete & & 83 \\
\hline 17 & 14 & $\mathrm{~F}$ & Sagittal & Complete & & 73 \\
\hline 18 & 15 & M & Sagittal & Complete & & 80 \\
\hline 19 & 15 & M & Sagittal & Complete & & 75 \\
\hline 20 & 18 & M & Sagittal, lambdoid & Complete & & 76 \\
\hline 21 & 18 & M & Sagittal & Complete & & 62 \\
\hline
\end{tabular}

Ant = anterior; pst $=$ posterior

* In sagittal sutures that were partially fused.

coronal and/or lambdoid sutures, 3 (75\%) were male and 1 (25\%) was female (Table 3).

Of the subjects with isolated sagittal suture fusion, 10 (59\%) had complete fusion, whereas 7 (41\%) had partial (but nearly complete) fusion (Table 4). When fusion was partial, the anterior end was minimally open in all 7 subjects; in 4 subjects, a short section of the posterior end was also open (Table 1). Of the subjects with complete fusion, $8(80 \%)$ were male and $2(20 \%)$ were female. Of the subjects with only partial fusion, $6(86 \%)$ were male and $1(14 \%)$ was female. There was no significant difference in the proportions of males and females with complete versus partial fusion ( $p=0.9999$; Table 4$)$. The mean age of subjects with complete fusion was 11.3 years, whereas that of the subjects with partial fusion was 5.6 years; the difference between the two was statistically significant ( $p$ $=0.0444$ ).

The mean age of subjects with isolated sagittal suture fusion was 8.9 years, whereas the mean age of subjects with medial fusion of adjacent sutures was 9.3 years; the difference between the two was not statistically significant $(\mathrm{p}=0.9641$; Table 3$)$.

All 4 subjects with medial fusion of coronal and/or lambdoid sutures had complete fusion of the sagittal suture (Table 1). In each of these subjects, fusion of the coronal and/or lambdoid sutures was partial and medial only.

Of all subjects with any sagittal suture fusion, 14 (67\%) had complete fusion and 7 (33\%) had partial fusion (Table 4). Of the subjects with complete sagittal suture fusion, 11 (79\%) were male and $3(21 \%)$ were female. In the entire group of 21 subjects, there was no significant difference in the proportion of males and females with complete versus partial sagittal suture fusion $(\mathrm{p}=0.9999)$. The mean age of all subjects with complete sagittal suture fusion was 10.7 years, whereas that of the subjects with partial fusion was 5.6 years, a difference that was statistically significant $(p$ $=0.0393$ ).

The mean age of all male subjects with any sagittal suture fusion was 8.4 years, whereas the mean age of females was 11.8 years; the difference between the two was not statistically significant $(\mathrm{p}=0.2078$; Table 2$)$. The mean age of males with isolated sagittal suture fusion and no medial fusion of adjacent sutures was 8.1 years, whereas the average age of females was 12.7 years; these ages were not significantly different $(\mathrm{p}=0.1454)$.

In all subjects with any sagittal suture fusion, the average CI was 78.0 (range 62-96). In subjects with isolated sagittal suture fusion, the average CI was 77.6 (range 6288); in subjects with medial fusion of adjacent sutures, the average CI was 81.3 (range 74-96; Table 3). The difference between the two was not significant $(\mathrm{p}=0.6526)$. In subjects with isolated sagittal suture fusion, subjects with complete fusion had an average CI of 75.8, whereas those with partial fusion had an average CI of 80.3; the differ- 

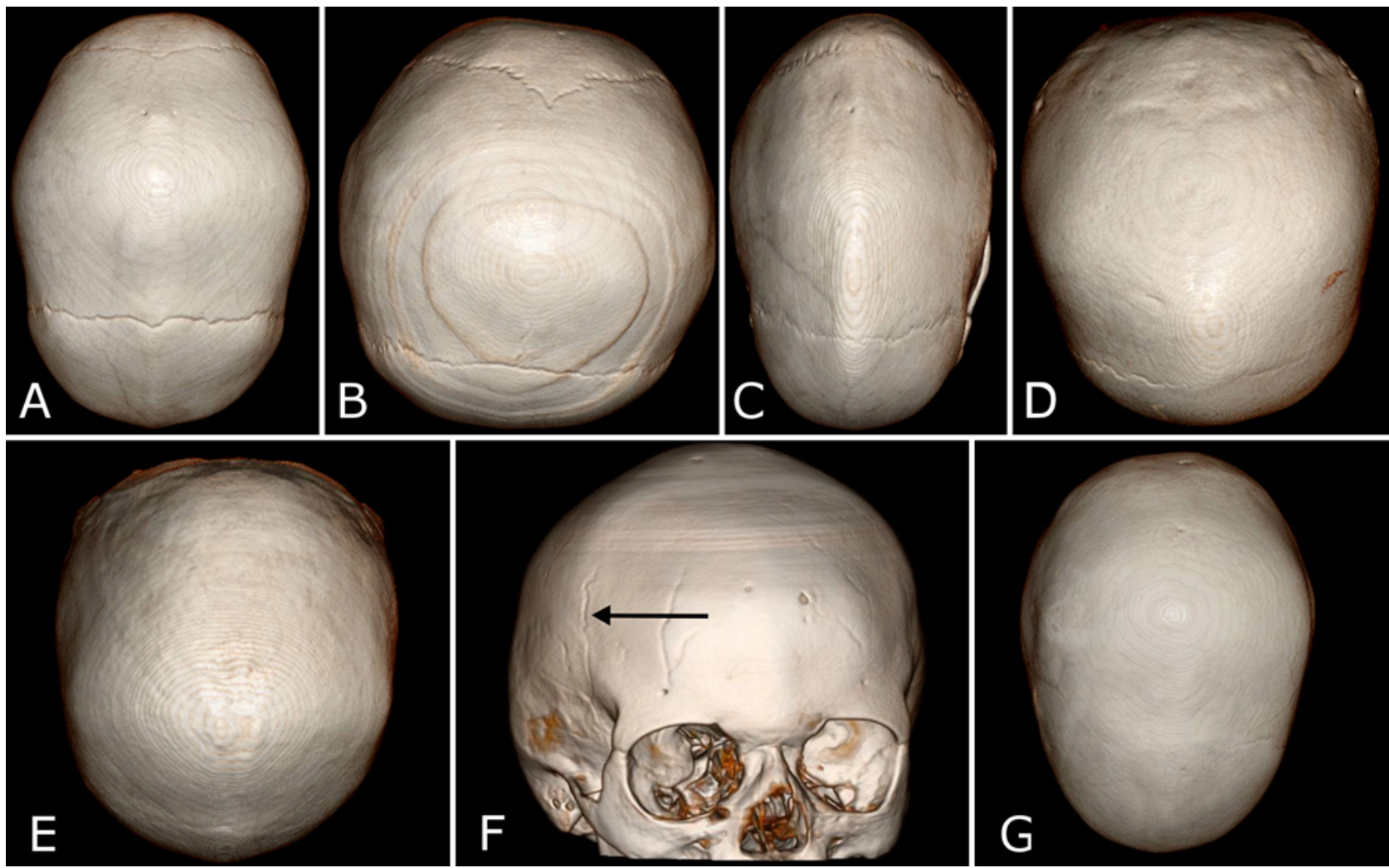

FIG. 1. Volume-rendered head CT scan reconstructions showing fusion or partial fusion of the sagittal suture with or without medial fusion of adjacent sutures. In each reconstruction except the one in panel $F$, anterior is rotated toward the bottom of the figure. A: Fusion of the sagittal suture only: complete fusion in a 27-year-old male, $\mathrm{Cl}=71$. B: Fusion of the sagittal suture only: partial fusion (anterior end of suture open) in a 24-month-old male, $\mathrm{Cl}=88$. C: Fusion of the sagittal suture only: complete fusion in an 18-year-old male, $\mathrm{Cl}=62$. D: Fusion of the entire sagittal and medial lambdoid sutures in a 9-year-old female, $\mathrm{Cl}=79$. $\mathrm{E}$ : Fusion of the entire sagittal and medial lambdoid sutures in an 18-year-old male, $\mathrm{Cl}=76$. F: Fusion of the entire sagittal and medial coronal sutures in a 5-year-old male, $\mathrm{Cl}=96$. A short segment of the lateral coronal suture remains open in this view (arrow). A similar section of coronal suture remains open on the opposite side of the calvaria. G: Fusion of the entire sagittal and medial lambdoid and coronal sutures in a 5-year-old male, $\mathrm{Cl}=74$. Figure is available in color online only.

ence between the two was not significant $(p=0.2018$; Table 4).

Coronal sutures were often bilaterally partially fused just above the pterion early in the 2 nd decade of life (Figs. $2 \mathrm{~B}-\mathrm{C}$ and 3 and Supplemental Table 2), and sporadic sutures were fused bilaterally in the same spots during the 1st decade. As the teenage years went on, fusion progressed symmetrically superiorly and medially and was present in progressively more subjects, with over $90 \%$ of coronal sutures partially fused by 18 years of age. However, none were ever completely fused even at 18 years. Of 60 partially fused coronal sutures, 57 had bilateral inferior fusion, 1 was fused inferiorly only on the right, and $2(3 \%)$ were fused medially. The latter 2 sutures were in subjects with sagittal suture fusion.

The metopic suture remained completely open in all subjects through 2 months of age (Fig. 4 and Supplemental Table 3). By 3 months, $29 \%$ were partially fused. Fusion was first seen just above the glabella and progressed inferiorly and superiorly as subjects aged (Fig. 2D). By 1 year of age and thereafter, nearly all metopic sutures were at least partially fused. However, completely open metopic sutures were sporadically found in subjects as old as 14 and 15 years (Figs. 2E-F and 4C). By 18 months, most metopic sutures were completely fused, although it was not unusual to find partially fused sutures even in the 3rd year of life. At 4 years and thereafter, 193 of 198 metopic sutures were completely fused (Fig. 4C); the exceptions were completely open. The last sections of the suture to fuse were just above the nasion and just in front of the anterior fontanel/bregma. The metopic suture was completely fused in all subjects with sagittal suture fusion. No metopic sutures were considered to be prematurely fused (i.e., no reconstruction was believed to demonstrate trigonocephaly).

\section{Discussion}

\section{Early and/or Atypical Fusion of the Sagittal, Lambdoid, and Coronal Sutures}

Twenty-one subjects in this study had fusion of most or all of the sagittal suture. None of the other 310 subjects had any sagittal suture fusion at all. Four subjects with sagittal suture fusion also had medial fusion of the coronal and/or lambdoid sutures $(0.9 \%$ of all lambdoid sutures, $0.6 \%$ of coronal). No other subjects had medial fusion of 

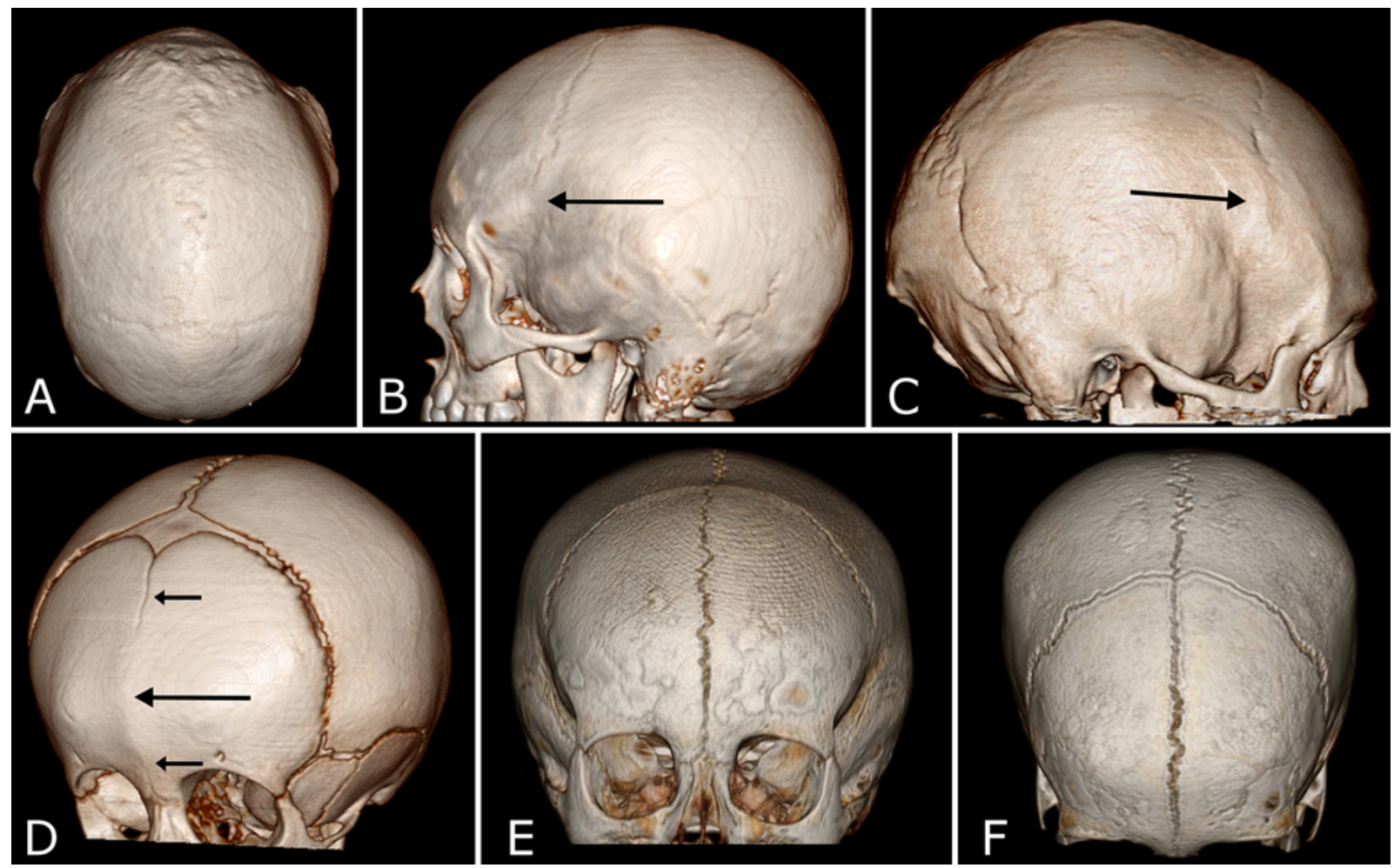

FIG. 2. Volume-rendered head CT scan reconstructions showing normal fusion patterns of major calvarial sutures. A: Extreme ridging of sagittal and lambdoid sutures in an 18-year-old female. Upon closer evaluation of the source images, these sutures were found to be open but narrow. They were presumed to be in the process of fusing, although no section of any suture is actually fused. The ridging does not involve the coronal suture. Anterior is rotated toward the bottom of the figure. B: Lateral fusion of the coronal suture (arrow), just above a fused sphenoparietal suture in an 11-year-old female. The coronal suture is fused in a similar location on the opposite side of the calvaria. C: Lateral fusion of the coronal suture (arrow), just above a fused sphenoparietal suture in a 17-year-old female. The coronal suture is fused in a similar location on the opposite side of the calvaria. D: Partially fused metopic suture in a 5-month-old male. Fusion (large arrow) has extended from just above the glabella inferiorly toward the nasion and superiorly toward an open anterior fontanel. Open sections of suture (small arrows) can be seen just superior to the nasofrontal suture and anterior to the anterior fontanel. These open sections of suture are less prominent than other open sutures and are probably in the process of fusing. E: Open metopic suture in an 8-year-old female. F: Open metopic suture in a 12-year-old male. Figure is available in color online only.

TABLE 2. Males versus females

\begin{tabular}{|c|c|c|c|c|c|c|}
\hline \multirow[b]{2}{*}{ Variable } & \multicolumn{3}{|c|}{ Subjects w/ Isolated Sagittal Suture Fusion $(n=17)$} & \multicolumn{3}{|c|}{$\begin{array}{l}\text { Subjects w/ Sagittal Suture Fusion w/ or w/o Medial Fusion } \\
\text { of Adjacent Sutures }(n=21)\end{array}$} \\
\hline & Male & Female & $\begin{array}{c}p \\
\text { Value }\end{array}$ & Male & Female & $\begin{array}{c}p \\
\text { Value }\end{array}$ \\
\hline No. (\%); adjusted \% & 14 (82); 72\% & $3(18) ; 28 \%$ & & $17(81) ; 70 \%$ & $4(19) ; 30 \%$ & \\
\hline $\begin{array}{l}\text { No. w/ multiple sutures } \\
\text { fused (\%) }\end{array}$ & & & & $3(18)$ & $1(25)$ & 0.9999 \\
\hline $\begin{array}{l}\text { Mean age in yrs (range); } \\
95 \% \mathrm{Cl}\end{array}$ & $8.1(2-18) ; 5.1-11.2$ & $12.7(12-14) ; 9.8-15.5$ & 0.1454 & $8.4(2-18) ; 5.6-11.2$ & $11.8(9-14) ; 8.5-15.0$ & 0.2078 \\
\hline $\begin{array}{l}\text { Complete vs partial fusion } \\
\text { of sagittal suture, no. } \\
(\%)\end{array}$ & $8(57)$ vs $6(43)$ & 2 (67) vs 1 (33) & 0.9999 & $11(65)$ vs $6(35)$ & 3 (75) vs 1 (25) & 0.9999 \\
\hline $\begin{array}{l}\text { Mean cephalic index } \\
\text { (range); } 95 \% \mathrm{Cl}\end{array}$ & 77.1 (62-88); 73.2-81.1 & $80.0(73-84) ; 64.9-95.1$ & 0.6573 & $78.0(62-96) ; 74.0-82.0$ & 79.8 (73-84); 71.8-87.7 & 0.6205 \\
\hline
\end{tabular}

$\mathrm{n}=$ number of subjects. 
TABLE 3. Isolated sagittal suture fusion versus fusion of sagittal and adjacent sutures

\begin{tabular}{lccc}
\hline \multicolumn{1}{c}{ Variable } & Isolated Sagittal Suture Fusion & Fusion of Sagittal \& Adjacent Sutures & $p$ Value \\
\hline No. (\%) & $17(81)$ & $4(19)$ & \\
\hline Complete vs partial fusion, no. (\%) & $10(59)$ vs $7(41)$ & $4(100)$ vs 0 (0) & 0.2550 \\
\hline Male sex, no. (\%); adjusted \% & $14(82) ; 72 \%$ & $3(75) ; 62 \%$ & 0.9999 \\
\hline Mean age in yrs (range); $95 \%$ Cl & $8.9(2-18) ; 6.3-11.5$ & $9.3(5-18) ; 0-19$ & 0.9641 \\
\hline Mean cephalic index (range); $95 \%$ Cl & $77.6(62-88) ; 74.2-81.1$ & $81.3(74-96) ; 65.3-97.2$ & 0.6526 \\
\hline
\end{tabular}

these sutures, and no others had fusion of the lambdoid suture at all. The sagittal and lambdoid sutures did not typically begin to fuse and the coronal suture did not typically fuse medially before 18 years of age.

By our reckoning, the aforementioned 21 subjects had early and/or atypical patterns of suture fusion. But did they have craniosynostosis? This is unclear, partly because none of them were examined clinically. Nevertheless, some of them clearly had scaphocephaly on their CT scans, both subjectively and per the CI (Fig. 1C), but others had normal shapes and CIs. Of the 17 subjects with sagittal suture fusion and no medial adjacent suture fusion, 6 had CIs greater than 80 and 4 had CIs that were 76-80 (10/17 total [59\%]), numbers commonly thought of as brachycephalic or mesocephalic. Did these 10 subjects just have unusually early but normal sagittal suture fusion? Or did they have premature fusion despite normal head shapes? This raises an interesting question regarding the definition of craniosynostosis; that is, whether craniosynostosis requires abnormal skull growth as traditionally thought or requires only premature sutural fusion. When craniosynostosis was first described in the 1800 s, it occurred in children with abnormal head growth. Nowadays, with the widespread availability of CT, there is the potential to find sutures fused unusually early or in unusual patterns in children with normal head growth. Should these cases be classified as craniosynostosis or normal? Longitudinal studies investigating how often head growth remains normal in such children may give us the answer. Regardless of the definition, when sutures are fused early or in un- usual patterns but skull growth is normal, surgery is not indicated. Until we have longitudinal studies, however, it would seem prudent to follow such children clinically to make sure head growth remains normal.

The incidence of craniosynostosis is often quoted to be approximately 1 in 2100-2325 live births $s^{3,9}$ and that of sagittal synostosis 1 in 5250-6667 subjects. ${ }^{3,10}$ However, the prevalence of early and/or unusual fusion of any major calvarial suture in our study was $6 \%$ and that of the sagittal suture alone was 5\%. Interestingly, Bolk in 1915 found that $2.5 \%$ of 1820 pediatric skulls had isolated "obliteration" of the sagittal suture. ${ }^{2}$ Bolk's numbers are also much higher than "modern" estimates of the incidence of craniosynostosis.

Why is fusion of the sagittal suture so prevalent in our study and Bolk's as compared to the accepted incidence of sagittal synostosis? The obvious reason may be correct: both studies included subjects with normal-not just abnormal-head shapes. A substantial number in Bolk's study were also "without any sign of scaphocephalic deformation." ${ }^{.17}$ Looking a little deeper, we found that both studies detect the prevalence of fusion over a range of pediatric ages versus the incidence in infants and toddlers. Perhaps some sagittal sutures fuse later in childhood than infancy, making the prevalence of fusion in all children higher than the incidence in infants. It is also likely that sutures that fuse later in childhood cause less skull deformity than those that are fused at birth because more skull growth has been completed, perhaps contributing to occult cases.

TABLE 4. Partial versus complete fusion

\begin{tabular}{|c|c|c|c|c|c|c|}
\hline \multirow[b]{2}{*}{ Variable } & \multicolumn{3}{|c|}{ Subjects w/ Isolated Sagittal Suture Fusion $(n=17)$} & \multicolumn{3}{|c|}{$\begin{array}{l}\text { Subjects w/ Sagittal Suture Fusion w/ or w/o Medial Fusion } \\
\text { of Adjacent Sutures }(n=21)\end{array}$} \\
\hline & Partial Sagittal Fusion & $\begin{array}{c}\text { Complete Sagittal } \\
\text { Fusion }\end{array}$ & $\begin{array}{c}p \\
\text { Value }\end{array}$ & Partial Sagittal Fusion & $\begin{array}{c}\text { Complete Sagittal } \\
\text { Fusion }\end{array}$ & $\begin{array}{c}\mathrm{p} \\
\text { Value }\end{array}$ \\
\hline No. $(\%)$ & $7(41)$ & $10(59)$ & & $7(33)$ & $14(67)$ & \\
\hline $\begin{array}{l}\text { No. w/ medial fusion of } \\
\text { adjacent sutures (\%) }\end{array}$ & & & & $0(0)$ & $4(29)$ & 0.2550 \\
\hline $\begin{array}{l}\text { Male sex, no. (\%); } \\
\text { adjusted \% }\end{array}$ & $6(86) ; 76 \%$ & $8(80) ; 68 \%$ & 0.9999 & $6(86) ; 76 \%$ & 11 (79); 66\% & 0.9999 \\
\hline $\begin{array}{l}\text { Mean age in yrs (range); } \\
95 \% \mathrm{Cl}^{*}\end{array}$ & $5.6(2-12) ; 2.0-9.1$ & $11.3(2-18) ; 8.0-14.6$ & 0.0444 & $5.6(2-12) ; 2.0-9.1$ & $10.7(2-18) ; 7.9-13.5$ & 0.0393 \\
\hline $\begin{array}{l}\text { Mean cephalic index } \\
\text { (range); } 95 \% \mathrm{Cl}\end{array}$ & 80.3 (72-88); 74.8-85.8 & 75.8 (62-87); 70.9-80.7 & 0.2018 & 80.3 (72-88); 74.8-85.8 & $77.4(62-96) ; 72.8-81.9$ & 0.3113 \\
\hline
\end{tabular}




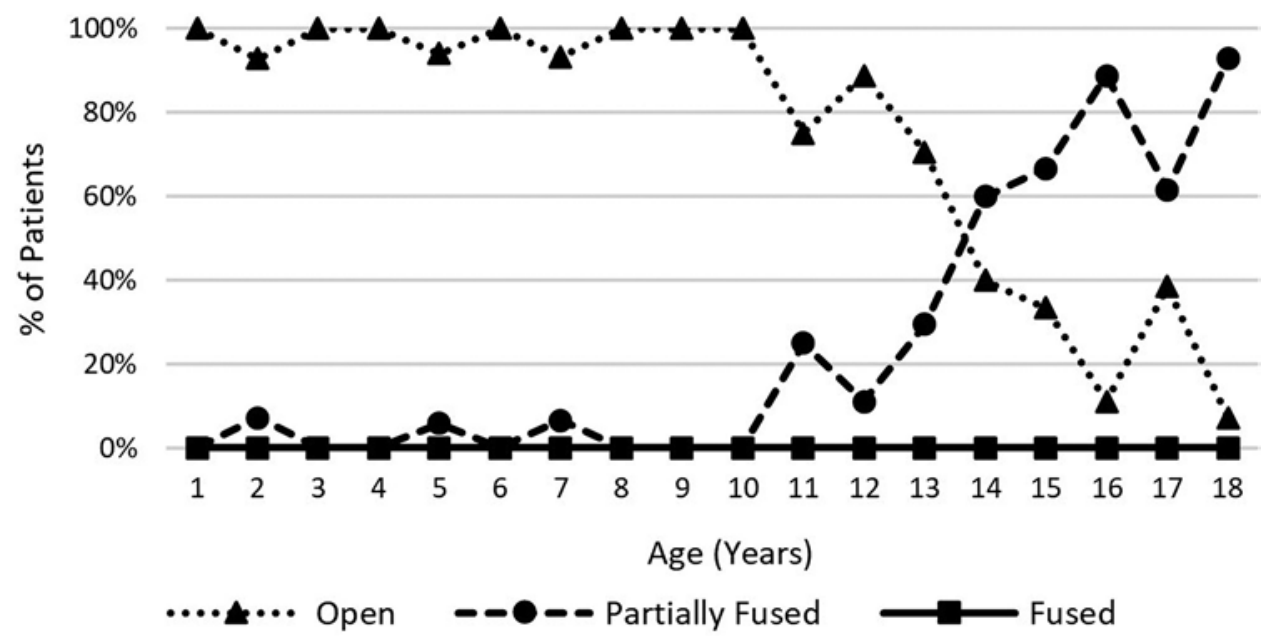

FIG. 3. Graph showing extent of coronal suture fusion (open, partially fused, and fused) versus age in years (1-18 years). Nearly all coronal sutures remained completely open until approximately 11 years of age, when a little more than $20 \%$ had started to fuse bilaterally. By 18 years of age, over $90 \%$ of sutures were partially fused. No coronal sutures were completely fused at any age in this study.

In 17 of 21 subjects with unusually early sagittal suture fusion in this study, no other sutures were fused early or in unusual patterns. Again, it is unclear whether these subjects had craniosynostosis. Still, the sagittal suture as the most common to fuse early or atypically is consistent with sagittal synostosis as the most common type of craniosynostosis. However, it is inconsistent with previously reported ratios of single-suture craniosynostosis, with most cases being sagittal, but significant minorities being metopic and coronal. .,4, $^{3-11}$ Against these 17 cases of isolated early sagittal suture fusion, there were no cases of isolated early metopic or coronal suture fusion (or lambdoid, for that matter). In Bolk's 1820 skulls, there were 47 cases of isolated sagittal suture fusion, 6 cases of isolated coronal fusion, and no cases of lambdoid fusion. ${ }^{2}$ Why there would be so many cases of early sagittal suture fusion and few to no cases of early fusion of other major sutures in these two studies is unclear.

The male/female ratio of our subjects with isolated sagittal suture fusion is consistent with sagittal synostosis being more common in males than females. ${ }^{3,10}$ After adjustment for the higher percentage of males than females in our series of pediatric trauma patients, $72 \%$ of our subjects with isolated sagittal suture fusion were male and $28 \%$ were female.

Of the fused sagittal sutures in our study, two-thirds were completely fused and one-third was partially fused. Each partially fused suture was open at one or both ends and fused in the middle, suggesting that fusion in all cases began somewhere between the two ends. All 7 partially fused sutures were open anteriorly, whereas only 4 were also open posteriorly, suggesting that fusion began somewhere in the posterior half of the suture. Authors of several older papers found that normal fusion of the sagittal suture begins posteriorly ${ }^{5}$ and, more specifically, at the obelion, the point between the parietal foramina..$^{14,18-20}$ Our findings are also consistent with early sagittal suture fusion beginning at the obelion.
Our subjects with complete sagittal suture fusion were significantly older than those with partial fusion, suggesting that fusion was a gradual process.

When the lambdoid and/or coronal sutures were fused early and/or atypically in this study, fusion was always medial and always contiguous with a completely fused sagittal suture. It was never solely lateral. Our cases of combined sagittal and lambdoid fusion were of the Mercedes-Benz type. ${ }^{13}$ This is all consistent with fusion spreading from the sagittal to adjacent parts of contiguous sutures.

\section{Normal Fusion of the Sagittal, Lambdoid, and Coronal Sutures}

Other than in the 21 subjects discussed above, no segments of any sagittal or lambdoid sutures were fused at all in this study. Seemingly, the sagittal and lambdoid sutures do not typically start to fuse until adulthood, in accordance with prevailing wisdom.

To our knowledge, fusion of the coronal suture commonly beginning before adulthood has never been described (although Harth et al. observed occasional fusion of segments of the coronal suture in the pediatric age range $^{6}$ ). We often found the coronal suture to be partially fused right above each pterion early in the 2 nd decade of life; fusion then proceeded superiorly and medially with advancing age. Fusion was bilateral and symmetric. In sporadic subjects, fusion began much earlier. Although fusion often extended quite far medially by age 18 , in no subject was the coronal suture completely fused. In the same series of CT reconstructions, we found that all sphenoparietal sutures were at least partially fused by 7 years of age. Thus, normal coronal suture fusion extends bilaterally from already-fusing sphenoparietal sutures.

Although fusion of the coronal suture commonly starting before adulthood has not been shown, fusion beginning laterally has. In a study of 82 skulls, Parsons and Box found many in which the coronal suture was fused below 

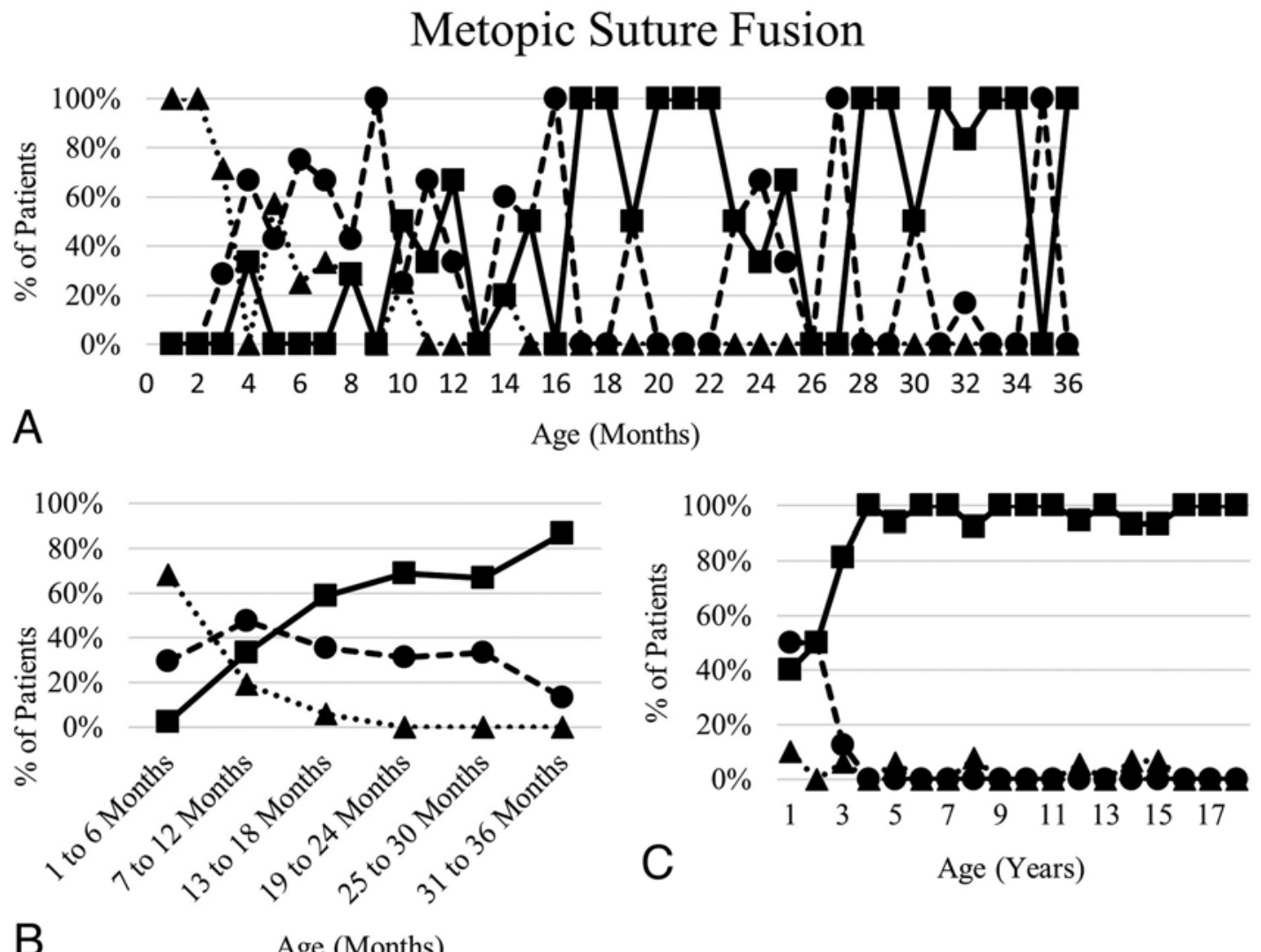

…. Open - - - Partially Fused $\rightarrow$-Fused

FIG. 4. Graphs showing extent of metopic suture fusion (open, partially fused, and fused) versus age by month (1-36 months, A), by 6 -month period (1-36 months, B), and by year (1-18 years, C). Up to 2 months of age, all metopic sutures remained open. By 3 months, nearly $30 \%$ were partially fused. By 1 year of age, virtually all metopic sutures were either partially or completely fused. By 18 months, most metopic sutures were completely fused, although it was not unusual to find partially open sutures even in the $3 \mathrm{rd}$ year of life. Rare sutures were still completely open as late as 14 and 15 years.

the stephanion but not above. ${ }^{14}$ In some, the sagittal suture was also partially fused at the obelion..$^{14}$ Of these 82 skulls, 4 were from subjects younger than 20 years old at death. Todd and Lyon examined the skulls of adult males in several studies ${ }^{18-21}$ and found that the "pars pterica" generally fuses before the "pars complicata" (midportion of the coronal suture) and the "pars bregmatica." Dwight also concluded that the coronal suture fuses first at the lower end. ${ }^{5}$

\section{Normal Fusion of the Metopic Suture}

The metopic suture has been reported to complete fusion by 6 to more than 40 months. ${ }^{1,12,15,22,23}$ Weinzweig et al. found that fusion proceeds from the nasion superiorly. ${ }^{23}$ Bajwa et al. found that fusion can begin anywhere in the inferior third of the suture. ${ }^{1}$ We found that the metopic suture remains open through 2 months of age. Fusion usually begins shortly thereafter just above the glabella and proceeds simultaneously inferiorly toward the nasion and superiorly toward the anterior fontanel/bregma. Although much of the suture is fused by 1 year of age, the inferior and superior ends often take a little longer.

Uncommonly, we found open metopic sutures in chil- dren as old as 14-15 years. Open metopic sutures after infancy have been described before. In 1543, before his pupil Falloppio first described sutures fusing, Vesalius described an adult suture pattern that included an open metopic suture. ${ }^{7}$ Parsons and Box's 82 skulls included 6 with open metopic sutures. ${ }^{14}$ The authors also mentioned that "it is sometimes taught ... that when the metopic suture fails to close at its usual time it is the last of all to be obliterated." Todd and Lyon also mention that "the metopic may remain open until late in life." " ${ }^{20}$ Dwight's skulls included two 82-year-olds with open "frontal sutures," and Bolk states that the "sutura frontalis" should remain in $6 \%$ of cases. ${ }^{2,5}$

\section{Clinical Implications}

Our findings have two major clinical implications: First, fusion of the sagittal suture in children and adolescents occurs more often than generally thought and may not be associated with abnormal skull growth. Children and adolescents with sagittal suture fusion and normal head growth do not need cranial surgery. Second, bilateral inferior coronal suture fusion is normal beginning early in the 2 nd dec- 
ade of life and may be an uncommon normal variant in the 1st decade. Children and adolescents with bilateral inferior coronal fusion do not need cranial surgery.

\section{Study Limitations}

First, except for the 21 subjects with sagittal suture fusion, the only records we reviewed for each case were initial trauma head CT scans. We assumed, probably wrongly, that our subjects had no preexisting conditions that would influence calvarial suture fusion. Likewise, we did not assess the prevalence of elevated intracranial pressure, visual deficit, chronic headaches, or developmental delay. This limited our ability to judge our subjects' need for surgery or to comment on any association between early suture fusion, elevated intracranial pressure, and developmental delay. ${ }^{8}$

We also assumed that the traumas suffered by our subjects did not cause any fused sutures to appear open on CT. We excluded sutures obviously involved by fractures, but we cannot be sure that other seemingly open sutures did not have subtle diastasis. We could have sought and reviewed negative $\mathrm{CT}$ scans of normal children and adolescents who had undergone CT scanning for reasons other than trauma. Instead, we utilized a large population of likely otherwise-normal subjects for whom volume-rendered reconstructions were readily available.

With different amounts of windowing, open sutures can appear more or less open, but fused sutures will not generally appear open. We were still extremely judicious with windowing when reviewing reconstructions. Also, volumerendered reconstructions typically visualize only the outer surface of the skull. However, the ectocranial and endocranial surfaces of cranial sutures actually fuse at different rates. ${ }^{2,14,16-21}$ In general, the radiographic extent of fusion may slightly differ from the physiological state of fusion.

Then there is the cross-sectional nature of the study. We did not follow individual subjects with periodic CT scans to track the progression of sutural fusion. Instead, we evaluated the extent of fusion on one scan per subject in subjects of different ages. We believe, however, that our study population is large enough that we can make valid inferences about the natural course of sutural fusion.

Still, it would have been nice to have more scans available to review. The number of available scans especially limited the number of metopic sutures evaluated at any particular month of age. However, we reviewed all scans available to us from the time that our hospital started routinely making volume-rendered skull reconstructions for trauma head CTs through the end of the institutional review board-approved study period. Soon, we may be able to review over 10 years of trauma CTs to corroborate our findings.

\section{Conclusions}

The sagittal and lambdoid sutures do not normally begin to fuse before 18 years of age. However, the sagittal suture fuses in children and adolescents more often than generally thought. The significance of this finding is unknown. Children and adolescents with sagittal suture fusion and normal skull growth do not need cranial surgery.
The coronal suture often begins to fuse inferiorly early during the 2 nd decade of life but does not usually complete fusion before 18 years of age. Children and adolescents with bilateral inferior coronal suture fusion do not need cranial surgery.

The metopic suture often starts to fuse by 3 months of age. Normal metopic sutures may not completely fuse until several years of age, if at all.

\section{References}

1. Bajwa M, Srinivasan D, Nishikawa H, Rodrigues D, Solanki G, White N: Normal fusion of the metopic suture. J Craniofac Surg 24:1201-1205, 2013

2. Bolk L: On the premature obliteration of sutures in the human skull. Am J Anat 17:495-523, 1915

3. Boulet SL, Rasmussen SA, Honein MA: A population-based study of craniosynostosis in metropolitan Atlanta, 19892003. Am J Med Genet A 146A:984-991, 2008

4. Cohen MM Jr: Editorial: perspectives on craniosynostosis. Am J Med Genet A 136A:313-326, 2005

5. Dwight T: The closure of cranial sutures as a sign of age. Boston Med Surg J 122:389-392, 1890

6. Harth S, Obert M, Ramsthaler F, Reuss C, Traupe H, Verhoff MA: Ossification degrees of cranial sutures determined with flat-panel computed tomography: narrowing the age estimate with extrema. J Forensic Sci 55:690-694, 2010

7. Hast MH, Garrison DH: Vesalius on the variability of the human skull: Book I Chapter V of De humani corporis fabrica. Clin Anatom 13:311-320, 2000

8. Hayward R, Britto J, Dunaway D, Jeelani O: Connecting raised intracranial pressure and cognitive delay in craniosynostosis: many assumptions, little evidence. J Neurosurg Pediatr 18:242-250, 2016

9. Lajeunie E, Le Merrer M, Bonaïti-Pellie C, Marchac D, Renier D: Genetic study of nonsyndromic coronal craniosynostosis. Am J Med Genet 55:500-504, 1995

10. Lajeunie E, Le Merrer M, Bonaïti-Pellie C, Marchac D, Renier D: Genetic study of scaphocephaly. Am J Med Genet 62:282-285, 1996

11. Lajeunie E, Le Merrer M, Marchac D, Renier D: Syndromal and nonsyndromal primary trigonocephaly: analysis of a series of 237 patients. Am J Med Genet 75:211-215, 1998

12. Lottering N, MacGregor DM, Alston CL, Watson D, Gregory LS: Introducing computed tomography standards for age estimation of modern Australian subadults using postnatal ossification timings of select cranial and cervical sites. J Forensic Sci 61 (Suppl 1):S39-S52, 2016

13. Moore MH, Abbott AH, Netherway DJ, Menard R, Hanieh A: Bilambdoid and posterior sagittal synostosis: the Mercedes Benz syndrome. J Craniofac Surg 9:417-422, 1998

14. Parsons FG, Box CR: The relation of the cranial sutures to age. J Anthropol Inst Lond 35:30-38, 1905

15. Pindrik J, Molenda J, Uribe-Cardenas R, Dorafshar AH, Ahn ES: Normative ranges of anthropometric cranial indices and metopic suture closure during infancy. J Neurosurg Pediatr 25:667-673, 2016

16. Sahni D, Jit I, Neelam, Sanjeev: Time of closure of cranial sutures in northwest Indian adults. Forensic Sci Int 148:199205, 2005

17. Singer R: Estimation of age from cranial suture closure. A report on its unreliability. J Forensic Med 1:52-59, 1953

18. Todd TW, Lyon DW Jr: Cranial suture closure. Its progress and age relationship. Part II.-Ectocranial closure in adult males of white stock. Am J Phys Anthropol 8:23-45, 1925

19. Todd TW, Lyon DW Jr: Cranial suture closure. Its progress and age relationship. Part III.-Endocranial closure in adult males of Negro stock. Am J Phys Anthropol 8:47-71, 1925 
20. Todd TW, Lyon DW Jr: Endocranial suture closure. Its progress and age relationship. Part I.-Adult males of white stock. Am J Phys Anthropol 7:325-384, 1924

21. Todd TW, Lyon DW Jr: Suture closure-Its progress and age relationship. Part IV.-Ectocranial closure in adult males of Negro stock. Am J Phys Anthropol 8:149-168, 1925

22. Vu HL, Panchal J, Parker EE, Levine NS, Francel P: The timing of physiologic closure of the metopic suture: a review of 159 patients using reconstructed 3D CT scans of the craniofacial region. J Craniofac Surg 12:527-532, 2001

23. Weinzweig J, Kirschner RE, Farley A, Reiss P, Hunter J, Whitaker LA, et al: Metopic synostosis: defining the temporal sequence of normal suture fusion and differentiating it from synostosis on the basis of computed tomography images. Plast Reconstr Surg 112:1211-1218, 2003

\section{Disclosures}

The authors report no conflict of interest concerning the materials or methods used in this study or the findings specified in this paper.

\section{Author Contributions}

Conception and design: Wilkinson, Stence, French. Acquisition of data: Wilkinson, Stence, Serrano, Graber, Batista-Silverman, Schmidt-Beuchat. Analysis and interpretation of data: Wilkinson, Stence, Graber, Schmidt-Beuchat, French. Drafting the article:
Wilkinson. Critically revising the article: all authors. Reviewed submitted version of manuscript: all authors. Approved the final version of the manuscript on behalf of all authors: Wilkinson. Statistical analysis: Graber. Administrative/technical/material support: Graber, Batista-Silverman. Study supervision: Wilkinson.

\section{Supplemental Information}

Online-Only Content

Supplemental material is available with the online version of the article.

Supplemental Tables 1-3. https://thejns.org/doi/suppl/10.3171/ 2019.11.PEDS1953.

\section{Previous Presentations}

Portions of this work were presented orally at the American Society of Pediatric Neurosurgeons 37th Annual Meeting held in Guanacaste, Costa Rica, in January 2014, and the 27th Annual Neurosurgery in the Rockies held in Beaver Creek, Colorado, in February 2014.

\section{Correspondence}

C. Corbett Wilkinson: Children's Hospital Colorado, University of Colorado Denver, Aurora, CO. charles.wilkinson@ childrenscolorado.org. 